All chests in which there was emphysema or reasonable suspicion of it, were omitted. The indices were: Tubercular patients (19), length 79.4 , breadth 78; normal individuals, length 85.5, breadth 72.5. In other words, the consumptive chest appears to be nearly 6 degrees both longer and rounder (deeper) than the normal, so that persistence of the fetal rounded or keeled type with a further reversion to the elongated ancestral type (both of which as already explained impair the efficiency in the erect position) would appear to co-exist at least with a predisposition to phthisis. If this is sustained by more extended measurements it will furnish a most valuable indication for climbing exercises and outdoor life in the chest measurements of children and young adults.

When we remember, in this connection, that the lung in the consumptive is really slightly more voluminous than normal, and that, as I have elsewhere suggested, the predilection of the tubercle bacillus for the lung is due to the ancestral recentness of the latter, constituting it the point of least sterility and resistance in the entire organism, it will be seen that the importance of this method of study is likely to be great. If it will help us to detect even a possible predisposition before the bacillus has gained a foothold it will bring us face to face with the hopeful and promising problem of prevention, instead of the discouraging and difficult one of cure. A month of tree climbing may be found worth a gallon of tuberculin.

\section{PHARMACOLOGY OF STROPHANTHUS.}

Presented in the Section on Materia Medica, Pharmacy and Therapeuties at the Forty-eighth Annual Meeting of the American Medical Association held at Philadelphia, June 1-4, 1897.

BY E. MARK HOUGHTON, M.D.

LECTURER ON EXPERIMENTAL PHARMACOLOGY, DETROIT CULIEGE OF MEDICINE.

DETROIT, MICH.

To Professor Fraser, more than to any one else, belongs the honor of having brought this drug to the notice of the medical profession, and for having determined in a general way its pharmacology and clinical value. I shall not discuss the botany, chemistry or therapeutics of the drug, as these questions have been treated in an appropriate manner by others. I have verified nearly all the important points made in this paper by experiments on animals, using for this purpose freshly isolated strophanthin, and 5 per cent. tinctures, tested by pharmacologic methods, prepared from drugs bought on the open market as strophanthus kombé. The strophanthin from this source is as active as the best strophanthin procurable, and more so than the majority of samples, which vary greatly in strength. Indeed, it was nearly one hundred times as strong as one lot which I examined, and which was claimed to be a chemically pure article.

In general, strophanthus, or rather the active constituent strophanthin, is primarily a nerve and muscle poison, which affects the functions of the various tissues of the animal body in a special manner, in therapeutic doses slowing the pulse, lengthening the systole and diastole, increasing the blood pressure, and tile efficiency of the cardiac muscle; and in toxic doses producing rapid paralysis of the heart.

Gley has shown that the local anesthetic action of strophanthin when brought in contact with the conjunctiva is about ten times as great as that of cocain, and that it persists much longer. Steinbaugh, from a long series of experiments on men and animals, concluded that this anesthetic action is not due to strophanthin, but to some other constituent which, when in a pure state, does not cause irritation or produce unpleasant after-effects. Hare and de Schweinitz, in attempting to confirm this work, concluded that Stein oaugh was mistaken and that the substance he used in his experiments was in reality strophanthin, which these authors declare to be a very dangerous drug in ophthalmic practice, since its application may be followed by inflammation and sometimes by ulceration.

Fraser, Boyd, Rovighi and others, claim that stro. phanthin has an antipyretic effect on patients suffer. ing from various diseases. Martin found the reverse. My experiments on dogs show that a slight lowering of temperature may be produced in normal animals. These investigators also report that the drug is a true cerebral sedutive. Their observations were made upon patients suffering from various diseases, and upon animals. In my studies sleep was one of the most constant symptoms noted in dogs, rabbits and guinea pigs under full doses of the drug. Brunton, Bau. beyer, Evans and others claim that strophanthus does not have a cumulative action when given for a long time. I have found that if very large doses are given to animals on successive days the second or third dose may be fatal. Especially is this true with rabbits, but generally doses very much greater than should ever be given for therapeutic purposes are well borne by dogs for a number of weeks in succession. In some instances the systern becomes more tolerant, as noted by Fraser.

Strophanthus is locally irritating and stimulating to the gastro-intestinal mucous membrane (Wood). It, however, produces much less disturbance to the stomach than does digitalis; in fact some authors claim that therapeutic doses act as a mild stomachic Clinical experience shows that in many cases strophanthus can be given where digitalis is contraindicated on account of the nausea and vomiting produced.

Strophanthus causes paralysis of striped and un. striped muscular tissue. The quickness with which this result is attained depends upon the concentration of the solution employed.

Like the other members of the digitalis series, strophanthus stops the frog's heart in systole, whether administered subcutaneously or locally. At first the pulsations become slower and more powerful; then the contraction changes to a peristaltic movement, the ventricles dilate less and less, and finally the ventricular cavity is completely obliterated, even when strong efforts are put forth by the auricles to expel their contents. Soon the auricles also cease to beat. This change occurs even when the inhibitory mechanism has been paralyzed by atropin; therefore, it must be due mainly to the action of the drug upon the muscular fibers themselves. The Strassburg school claim that the systolic condition is due to increased elasticity of the muscle fibers. The work of Roy, however, has thrown considerable doubt on this point, while Cushny thinks the ultimate systolio condition is probably due to increased contractility. The whole question is far from settled at the present time.

Since strophanthus is generally used in the treatment of diseases of the circulation, a consideration of its action on the heart and blood vessels of animals is of chief importance. The normal circulation of the 
animal body, with its many variations that may influ. ence the blood supply to this or that particular organ, is maintained mainly by the action of the heart and the muscular fibers of the arterioles under the influ. ence of the central nervous system, which in turn is regulated by the different afferent impulses or direct influences reaching it. To understand the action of strophanthus upon these factors is to rationalize its therapeutic employment in those cases where the organism is suffering because of some pathologic condition of its vascular mechanism.

Pharmacologists are very well agreed that digitalis, strophanthus, lily of the valley, and the other members of the group have the same qualitative action upon the circulation, but the quantitative relation of the various drugs of the series to the different physiologic factors has been only partially worked out. Most of the work has been done on strophanthus and digitalis. When these various quantitative relations have been settled we shall be able to apply one or another of these remedies, as the case may be, with the greatest certainty according to the pathologic conditions presented in the patient.

A careful study of the papers of the early experimenters on digitalis shows that section of the vagus does not entirely prevent slowing of the heart, but if atropin be given the rhythm becomes normal; consequently the decreased pulse rate when therapeutic doses are employed is mainly due to the stimulation of the vagus centers, and partially to irritation of the peripheral endings of the vagus in the heart. Considerable dispute has arisen as to whether the behavior of the vagus be not due simply to the increased supply of blood to its medullary centers, but it seems to be well established that the drug has a direct action on these centers.

Brunton and others claim that the quickening of the pulse in the second stage is caused by paralysis of the vagus ends, while Schmiedeberg, Cushny and Boehn have shown that it is probably due mainly to the increased irritability of the muscular fibers of the heart. Generally with the slowing of the rhythm the most constant result noted is the increase in blood pressure; but this does not always occur, especially where there is extreme slowing. Brunton and many others regard the increase in pressure after administration of digitalis as due mainly to the constricting action of this drug upon the peripheral circulation, but confess that strophanthus does not narrow the caliber of the arterioles.

Fraser found, by perfusion experiments with strophanthus, that exceedingly large doses of the drug are required to produce even transient constriction of the vessels. Popper claims that with strophanthus the vessels are redder than before. Delsaux, Bradford and Philipps found that the volume of the kidney remains unchanged when studied with the oncometer.

My experiments on various animals convince me that no constriction of the vessels is produced by therapeutic doses of strophanthus. The increased blood pressure must be due mainly to direct action upon the heart.

Popper claims that venous pressure falls as the arterial pressure rises. Little experimental work has been done to prove this point.

Popper and Knoll find that the blood pressure has less influence on the pulmonary circulation than on the systemic circulation. Gley found that curare dues not alter the action of strophanthus on the blood pressure, which consequently does not depend on changes in respiration.

Rolleston claims that the maximum pressure in the left ventricle and the output of the heart are increased and the circulation quickened by strophanthin.

Roy and Adami found that strophanthus at first increased the force and efficiency of the papillary muscles, with only slight effect on the walls of the ventricles; large doses quickly paralyze these muscles, having less action on the ventricular walls. Dissociated contractions of the ventricles and auricles occur before this final paralysis.

The recent exhaustive paper of Cushny on the digitalis series has very largely cleared up the question of the relations of increased muscular irritability and central peripheral vagus action. $\mathrm{He}$ employed the myocardiagraph, which records the relative distance between two points on the heart, and the cardiometer, which measures the volume of the heart at successive stages, and consequently registers the amount of blood ejected by this organ. These two instruments were of the form devised by Roy and Adami. Cushny divides the action of the drug into: 1 . The vagus stage, which is characterized by the results of the vagus stimulation and the direct action on the heart muscle itself. 2. The periodic stage, which begins with acceleration of the ventricles. He examined the movements of the four chambers independently, and also determined their relation to each other when. under the influence of strophanthus, employing the myocardiagraph. When small quantities of the drug are administered intravenously there is marked slowing of the rhythm of the heart, the ventricle dilates more fully, and systole is more perfect. The sequence of these changes is not always the same. The slowing and increased contraction are the most constant manifestations noted. Cushny agrees with Ackerman that the former is due to inhibitory action of the vagus, and claims that the increased dilatation is also due to inhibition. Neither slowing nor dilatation occurs when atropin is administered, but this substance has no influence on the increased contractility, which must consequently be due to the action of strophan. thus on the muscle fibers themselves.

Without atropin the rhythm is decreased, diastole and systole are more pronounced, and the blood pressure is raised. Tracings taken on fast moving drums show the same, also that the slowing is mainly due to the lengthened diastole, although the pause in systole is somewhat greater. The slowing and increased diastole are the same as when the vagus is stimulated. The time of the relaxations and contractions remains the same as before in the normal animal.

The muscular action of strophanthus is the same on the auricle as on the ventricle, but is less apparent and may be concealed, owing to the greater influence of the vagus, exerting more opposition to the increased muscular iritability. Shortly, if the drug is pushed, what may be called the pause phase begins to manifest itself and the rhythm, which heretofore has been the same in the auricles and ventricles becomes changed. Irregularity and skipped beats may occur in either chamber first, after which the rhythm of the superior and inferior portions of the heart exhibits wide divergence. The systole and diastole become more and more incomplete, until finally considerable pauses are noticed, especially in the auricle. The two sides of the heart after a few intermittent beats begin suddenly, showing a much accelerated rhythm, but 
the rhythm of the auricles and that of the ventricles is entirely independent, and impulses no longer pass from one to the other.

By careful estimation of the amount of blood passing through the heart as recorded by the cardiometer during the first stage of strophanthus action, Cushny maintains that, "the contraction volume or output of the ventricle per beat is uniformly much increased, whether slowing of the rhythm is marked or not. This is, of course, due to increased systole, augmented in some cases by increased diastole of the ventricles." The amount of blood expelled per unit of time varies according to the amount of slowing produced. Where the rhythm is only slightly lessened the output is increased, but if it is greatly retarded the output is below normal.

This increased work must be due to increased muscular action, since Roy and Adami find that the heart ejects less blood when the vagus is stimulated. The increased dilatation of the ventricles due to vagus stimulation, helps to offset the slowing. If the nervous mechanism is paralyzed with atropin the contraction volume is greater, owing to more perfect systole and the output is uniformly increased.

Blood pressure is raised during the first stage, even before a change in the heart is noticed. In general, during the first stage analogous results are shown by the myocardiagraph, cardiometer and kymograph, slowing and increased work; if atropin is used increased work without slowing.

I have dwelt at length on the primary action of strophan thus because it is never desirable to go beyond this stage under any condition in the therapeutic application of the remedy. In fact it is not wise to produce more than a moderate degree of the first stage, as better filling of the vessels is secured when the rhythm is but moderately decreased.

At the close of the first stage, as already mentioned, the ventricular and auricular contractions become slower and slower, and may occasionally skip; soon, however, the irritability of the muscle becomes so much increased that the restraint of the vagus is thrown off and the ventricles usually first, soon followed by the auricles, show an accelerated, very irregular rhythm, the ventricles beating together, and the auricles also having the same rhythm, while the normal sequence of the respective contractions is entirely destroyed. The inhibitory mechanism still acts, but is entirely insufficient to control the exaggerated muscular activity. The individual beats of the different chambers are very irregular and the amount of work is greatly lessened; soon the spasmodic effects of the organ cease, generally in the auricles first, in a position somewhat nearer diastole than systole; then follow delirium cordis and complete dilatation.

Knoll, working with a less perfect myocardiagraph than that devised by Roy and Adami, obtained results which correspond quite accurately with those obtained by Cushny. Neither Cushny nor Knoll has yet completed their observations on the action of the heart during the second stage. During the second stage there is generally present an increased excitability of the central nervous system, and violent movements of the respiratory and other muscles occur, the animel appearing much as though under the influence of one of the convulsive poisons.

It has been found from extensive experiments that diuresis is one of the most constant results noted when strophanthus is administered to healthy individuals
(Semoine) or to patients suffering from various cardiac lesions (Fraser, Semoine, Wood and others). The diuresis may be due entirely to increased blood pressure, to direct renal action (Wood), or to a combination of the two. The action of strophanthus is very much more rapid, and the drug is eliminated from the system more quickly than is the case of digitalis, presumably because of the greater solubility of its active constituent strophanthin, which is probably excreted in the urine (Wood).

During the past two years with a view of ultimately being able to standardize all the various preparations of the digitalis and strophanthus series by physiologic methods-since they can not be assayed by chemic means-I have examined hundreds of samples of crude drug and active constituents, as purchased on the open market or procured from private sources. The task is indeed a difficult one, owing to the small amount of quantative work that has been done. Sufficient data have already accumulated to convince me that the task of perfecting pharmacologic methods for standardizing all those powerful drugs and chemicals which can not be assayed by other methods is not a hopeless one. Owing to the ready solubility of strophanthin, the preparations of strophanthus are perhaps the most easily standardized of the heart tonics.

As already stated the strophanthin on the market varies greatly in strength. The same is true of the crude drug. As physicians, I believe the time has come when we should demand at least a qualitative pharmacologic examination of these drugs before they are made up into pills, tablets, tinctures, fluid extracts, etc.

The sphygmographic work of Fraser, Paschkis, Zerner and especially Wilcox, shows that strophanthus produces the same effects on the circulatory apparatus of man as upon that of other mammals. The fatal dose of strophanthus varies for different groups of animals. Reusing and others claim that strophanthus is at least twenty times as strong as digitalis. Fraser claims it is 300 times as strong. I have found one sample of strophanthin sufficiently powerful to kill medium-sized frogs in doses of 1-7000 of a grain. For therapeutic use I believe 1.300 to $1-200$ grain of strophanthin, and from 3 to 5 drops of a 5 per cent. tincture to be the correct dose.

Briefly summarizing the pharmacology of strophanthus we may say that its chief action is upon the nervous mechanism controlling the action of the heart, and upon the heart muscle itself, lessening pulse rate, increasing the blood pressure and augmenting the work of the heart; without causing constriction of the arterioles or any special action on the vasomotor mechanism; the diuresis and other important results being due mainly to improved circulation.

Its special advantages over digitalis may be briefly stated as follows: It does not produce gastric disturbances, and does not show cumulative action. The constriction of the vessels by digitalis may be a source of great danger owing to the extra strain thrown on the ventricle, especially in fatty heart. Strophanthus has no such tendency. It acts quicker and with greater certainty. Owing to its ready solubility it is better for hypodermatic administration, and the strength of its preparations can be more easily standardized.

Let us have a Department of Public Health! 